\title{
Recherche et mouvement associatif : des ponts à construire
}

Nos lecteurs le savent, une réflexion est en cours au sein du comité de rédaction, qui a comme objet d'adapter notre revue à son contexte actuel à partir des enseignements qui peuvent être tirés de ses dix-huit années d'existence. En témoignent d'ores et déjà les modifications apportées à sa structure et à sa maquette. C'est pour accompagner cette réflexion que l'association Natures Sciences Sociétés - Dialogues a pris l'initiative d'organiser, en partenariat avec six autres associations, un Forum des associations intitulé "Repenser le développement : la société civile s'engage », qui s'est déroulé les 20, 21 et 22 janvier 2011 à la Cité internationale universitaire de Paris et qui a été un intense moment de réflexion partagée entre une centaine d'associations ( $c f$. http://www. nss-dialogues.fr/Forum-des-associations-01-2011).

En quoi une revue scientifique comme NSS a-t-elle à voir avec un rassemblement de cette nature? Cette question appelle une réponse en deux volets : le premier porte sur la référence au développement durable, car le forum a montré qu'elle s'avérait centrale pour organiser le débat sur la crise actuelle; le second concerne le rapport entre démarche de recherche et mouvement associatif.

NSS ne s'est jamais explicitement placée sous la bannière du développement durable. Bien qu'étant directement le produit de la montée en puissance de la question environnementale, elle ne s'était d'ailleurs pas davantage, ni dans son titre et son sous-titre ni dans son projet éditorial, référée au terme " environnement », qui prévalait dans le discours ambiant de l'époque où elle a été créée. Le choix qui a alors été fait dans leurs formulations marquait la volonté de l'identifier avant tout par une démarche scientifique. Le projet était bien de contribuer conjointement à construire un champ de recherche en soi (les rapports entre "sociétés » et «natures ») et à expérimenter une autre façon de faire de la recherche tenant compte du statut hybride des objets étudiés et des questions posées ( "l'interdisciplinarité »). Cette posture était choisie comme moyen de se donner le recul indispensable pour pouvoir clarifier, en en explicitant les raisons et les enjeux, les discours ambiants (et contradictoires, si ce n'est polémiques) issus tant de divers groupes sociaux et du politique que des différents secteurs de la recherche, sur les questions mettant en jeu cette dualité. Et cela dans le but de dégager des voies susceptibles de faire avancer la réflexion et l'action.

Une approche aussi transversale est applicable à de nombreux domaines. La revue s'en fait l'écho dans la mesure de ses possibilités. Il n'en demeure pas moins que ce projet éditorial ne pouvait manquer de la mettre prioritairement aux prises avec la notion de développement durable, compte tenu de l'omniprésence de celle-ci. Les textes qui y sont publiés illustrent effectivement l'influence que cette notion a sur les chercheurs. Cela traduit son intérêt sur le plan heuristique, qu'elle tire de la combinaison entre une perspective constructiviste et sa vertu à mettre le chercheur dans une posture d'analyse à la fois critique (sur la base des valeurs qu'elle implique), multidimensionnelle, contextualisée et incluant l'action. Elle s'avère ainsi un outil particulièrement adapté à l'objectif scientifique de NSS. De ce fait, la revue est, volens nolens, un des lieux de la réflexion sur cette notion et de sa construction en vue de l'action; et un lieu d'un intérêt tout particulier, dans la mesure où il témoigne en temps réel des apports à la fois critiques et constructifs d'une recherche soucieuse de la considérer dans toutes ses dimensions, si ce n'est dans sa globalité. Ambition dont il est important de prendre conscience afin d'en tirer les conséquences.

Une des conséquences notables est - et c'est là le second volet de la réponse à la question posée - de mettre en évidence le fait que ce projet éditorial fait partie de l'ample mouvement de réflexion et d'actions porté par les très nombreuses associations qui œuvrent pour un développement durable. Il a cette spécificité de viser à formaliser cette réflexion et ces actions et à les éprouver sur un plan scientifique. Force est de constater que la revue est coupée actuellement de ce mouvement. 
N'a-t-elle pas maintenant un défi à relever dans le droit fil de son exigence initiale? En effet, cette coupure la met en contradiction avec une des dimensions majeures de son ambition novatrice, à savoir le réexamen des rapports sciences-sociétés qu'implique l'idée d'un développement durable, avec ses prolongements sur des questions aussi centrales aujourd'hui que celles ayant trait aux conditions de production de la connaissance, aux rapports entre savoirs et aux modalités des rapports de la recherche à l'action. Les associations ont une expérience particulièrement riche à faire valoir en la matière. L'ouverture sur elles offre l'opportunité de mettre en œuvre le partenariat sociétal recherché, de mobiliser ainsi l'expérience sociale, culturelle, politique qu'elles acquièrent à travers leurs actions et de s'interroger sur les conditions de sa mise en problématique sur un plan scientifique.

Cette ouverture se justifie d'autant plus que, si les associations en cause peuvent être une ressource pour la recherche, l'inverse est aussi vrai, car leurs réflexions et leurs actions ont comme point de départ des résultats de celle-ci. La pertinence de l'action associative suppose un dialogue permanent avec elle. Cela a été clairement illustré par le Forum des associations, qui a été un espace original de dialogue entre militants associatifs et scientifiques. La notion de développement durable, à la fois incontournable et très débattue, s'y est avérée un excellent outil de controverse.

La question est donc maintenant posée à la revue de l'opportunité pour elle de tirer profit de cette manifestation et de la façon de le faire. Elle peut être formulée ainsi : Comment intégrer les discours et les pratiques associatives dans une démarche scientifique? $\mathrm{Ou}$ bien, autre formulation : Comment faire d'associations des partenaires en recherche (en l'occurrence, à travers la conduite d'une revue scientifique) ? C'est en fait déjà à cette question que NSS essaie de répondre dans le domaine qui est le sien (la recherche), en militant (le terme est approprié) pour des pratiques de recherche différentes de celles qui ont habituellement cours. Elle le fait en travaillant à donner à ces pratiques novatrices des formes qui les rendent recevables dans le champ scientifique, car là est l'enjeu pour une recherche active en prise sur les problèmes posés aux sociétés. Faire d'elle un outil pour pérenniser ce dialogue n'est-il pas la voie à suivre pour que les questions vives de l'action et les questions vives de la recherche s'entraînent et se confortent les unes et les autres, tout en laissant à chacune d'elles les marges d'autonomie qu'exigent leurs finalités différentes?

La revue ferait ainsi un pas de plus dans le sens de son projet : le pas qui consiste à intégrer la « société civile organisée » à la recherche sur le développement durable. Elle montrerait alors d'elle-même le visage plus accompli d'un lieu où se capitalise, à travers une synergie renforcée entre recherche et action, la réflexion sociétale autour du projet de «repenser le développement ». 